\title{
LE SESSE DEL LAGO DI GARDA
}

\author{
Parte IV \\ OSCILLAZIONI LIBERE DEL BACINO ORIENTALE \\ P. Caloi - M. C. Spadea
}

I. - Ẽn bacino esteso e dalla forma complessa come quello del Lago di Garda, non può evidentemente esaurire le proprie libere oscillazioni a quelle che lo interessano nella sua totaliti. Anche i suoi golfi e parti determinate comunque limitate dalle sponde, possono essere sede di oscillazioni proprie. Albliamo visto cosi che libere oscillazioni possono avere la parte seltentrionale del Garda, il Golfo di Salò, il Golfo di Desenzano nonché quelle parli del Lago caratterizzate da un lento procerlere dello zoccolo verso le regioni più profonde. Queste ultime parti danno origine alle cosi delle "self-seiches", che risullano particolamente sensibili nel golfo di Desenzano.

Una trattazione a parte meritava il bacino orientale del Garda Chacino Peschiera-Garda), che, solto certi aspelti, forma un bacino a sé. Infalti l'esistenza di una dorsale suluacquea (dai pescatori chiamata "linea dei monti $"$ l clie da Punta Sirmione porta a Punta S. Vigilio, viene a fare del bacino Peschiera-Garda un bacino con caratteristiche proprie, con propri movimenti. Oserviamo ancora che tale hacino ha una profondita media molto piccola e che pertanto sareblue capace, qualora le condizioni idrodinamiche lo consentisero. di lunghissine oscillazioni proprie.

Lo studio delle oscillazioni di questo bacino si presentava pertanlo, tutl'altro che agevole. Le osservazioni mostravano l'esistenza di tutla una serie di oscillazioni, registrate a Peschiera, che da periodi di $30^{\mathrm{m}}$ ca. vanno a periodi di pochi minuti.

In questo lavoro ci siamo proposti di giustificare tali oscillazioni nel senso fisico e di spiegare le loro principali caratteristiche, intimamente legate alla forma del bacino stesso, alla sua piccola profondia ed al fatto di essere esso collegato con un bacino molto più esteso. ma nello stesso tempo noterolmente più profondo. 


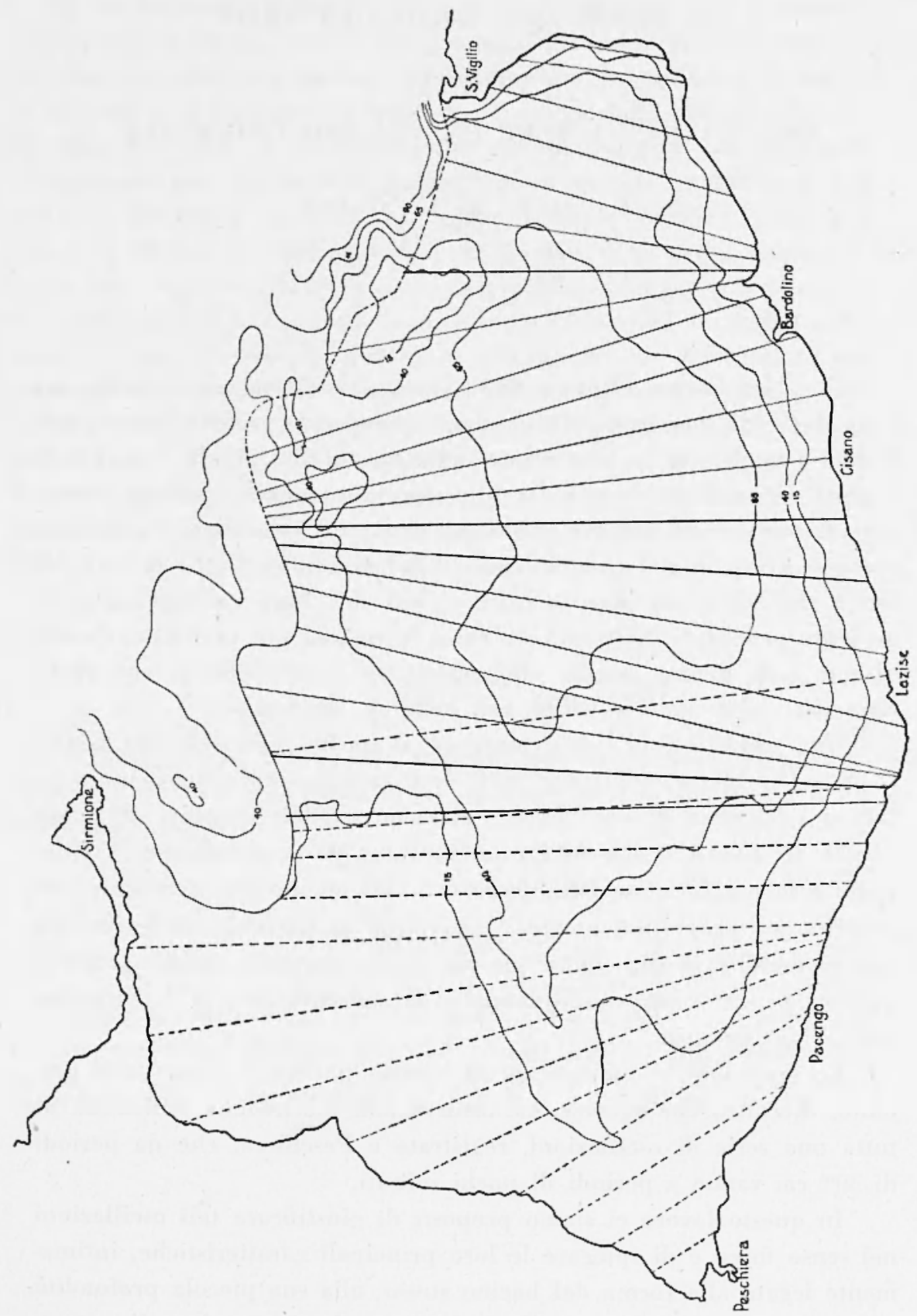

Fig. 1 
Tabella 1

\begin{tabular}{|c|c|c|c|c|c|c|c|c|c|c|c|c|c|c|c|c|c|c|c|}
\hline 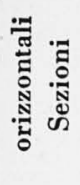 & $\underset{\text { distanza }}{\mathbf{m}}$ & $\begin{array}{c}S(x) \\
\text { Sezioni } \\
\text { verticali } \\
\mathbf{k m}^{2}\end{array}$ & 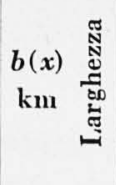 & $\begin{array}{c}V(x) \\
\mathbf{k m}^{2} \\
\text { Singole }\end{array}$ & 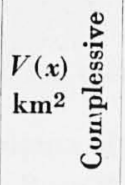 & $z=\frac{v}{a}$ & $z^{2}$ & $(1-z)$ & $(1--z)^{2}$ & $z^{2}(1-z)^{2}$ & $\sigma(z)$ & $\frac{2(1--z)^{2}}{G(z)}$ & $\Delta z$ & $\frac{z^{2}\left(1-z^{2}\right)}{\sigma(z)}=$ & $M z$ & $M z^{2}$ & $M z^{3}$ & $M z^{t}$ & \\
\hline $\begin{array}{r}0 \\
1 \\
2 \\
3 \\
4 \\
5 \\
6 \\
7 \\
8 \\
9 \\
10 \\
11 \\
12 \\
13\end{array}$ & $\begin{array}{r}0 \\
500 \\
1000 \\
1500 \\
2000 \\
2500 \\
3000 \\
3500 \\
4000 \\
4500 \\
5000 \\
5500 \\
6000 \\
6500\end{array}$ & $\begin{array}{l}0 \\
.0892 \\
.2476 \\
.4000 \\
.5270 \\
.6105 \\
.6400 \\
.6965 \\
.6633 \\
.5990 \\
.5583 \\
.5015 \\
.4433 \\
.2966\end{array}$ & $\begin{array}{r}0 \\
6,25 \\
7,70 \\
10,80 \\
12,75 \\
14,55 \\
14,25 \\
13,85 \\
13,87 \\
11,15 \\
10,70 \\
10,45 \\
9,40 \\
8,95\end{array}$ & $\begin{array}{c}0 \\
2,81 \\
3,45 \\
5,13 \\
6,30 \\
6,97 \\
9,14 \\
7,69 \\
9,32 \\
7,33 \\
6,04 \\
5,99 \\
5,69 \\
5,55\end{array}$ & $\begin{array}{c}0 \\
2,81 \\
6,26 \\
11,39 \\
17,69 \\
24,66 \\
33,80 \\
41,49 \\
50,81 \\
58,14 \\
64,18 \\
70,17 \\
75,80 \\
81,41\end{array}$ & $\begin{array}{l}0 \\
.03452 \\
.07689 \\
.13991 \\
.21729 \\
.30291 \\
.41518 \\
.50964 \\
.62412 \\
.71416 \\
.78835 \\
.86193 \\
.93183 \\
1,00000\end{array}$ & $\begin{array}{l}0 \\
.001192 \\
.005912 \\
.019575 \\
.047215 \\
.091754 \\
.172374 \\
.259733 \\
.389626 \\
.510224 \\
.621496 \\
.742923 \\
.868307 \\
1,00000\end{array}$ & $\begin{array}{l}0 \\
.96458 \\
.92311 \\
.86009 \\
.78271 \\
.69709 \\
.58482 \\
.49036 \\
.37588 \\
.28584 \\
.21165 \\
.13807 \\
.06817 \\
0\end{array}$ & $\begin{array}{l}0 \\
.932152 \\
.852132 \\
.739755 \\
.612635 \\
.485934 \\
.342014 \\
.240453 \\
.141286 \\
.081704 \\
.044796 \\
.019063 \\
.004647 \\
0\end{array}$ & $\begin{array}{l}0 \\
.011111 \\
.050370 \\
.144807 \\
.289256 \\
.445864 \\
.589543 \\
.624536 \\
.550346 \\
.416710 \\
.278405 \\
.141623 \\
.040350 \\
0\end{array}$ & $\begin{array}{l}0 \\
.000557 \\
.001906 \\
.004320 \\
.006719 \\
.008883 \\
.009120 \\
.009646 \\
.009200 \\
.006679 \\
005974 \\
.005241 \\
.004167 \\
.002655\end{array}$ & $\begin{array}{c}0 \\
19,930 \\
26,424 \\
33,520 \\
43,049 \\
50,194 \\
64,643 \\
64,742 \\
59,820 \\
62,393 \\
46,604 \\
27,024 \\
9.683 \\
0\end{array}$ & $\begin{array}{l}0 \\
.03452 \\
.04237 \\
.09754 \\
.11975 \\
.18316 \\
.23202 \\
.27762 \\
.34650 \\
.36766 \\
.42069 \\
.44124 \\
.49059 \\
.50941\end{array}$ & $\begin{array}{c}0 \\
.68798 \\
1,11958 \\
3,26954 \\
5,15512 \\
9,1953 \\
14,99847 \\
17,97367 \\
20,72763 \\
22,93941 \\
19,60584 \\
11,92407 \\
4,75038 \\
0\end{array}$ & \begin{tabular}{c}
\multicolumn{1}{c}{0} \\
.02375 \\
.08608 \\
.45744 \\
1,12016 \\
2,78481 \\
6,22706 \\
9,16010 \\
12,93653 \\
16.38241 \\
15,45626 \\
10,27771 \\
4,42655 \\
0
\end{tabular} & $\begin{array}{c}0 \\
.00082 \\
.00662 \\
.06400 \\
.24340 \\
.84354 \\
2,58535 \\
4,66835 \\
8,07395 \\
11,69965 \\
12,18495 \\
8,85866 \\
4,12479 \\
0\end{array}$ & $\begin{array}{c}0 \\
.000028 \\
.060509 \\
.008954 \\
.052886 \\
.255517 \\
1.073386 \\
2.379178 \\
5.039114 \\
8,355422 \\
9,606005 \\
7,635545 \\
3,843603 \\
0\end{array}$ & $\begin{array}{l}0 \\
.000001 \\
.000039 \\
.000688 \\
.011492 \\
.007399 \\
.445648 \\
1,212524 \\
3,145012 \\
5,97108 \\
7,572894 \\
6,581305 \\
3,581584 \\
0\end{array}$ & $\begin{array}{l}\mathrm{I}_{0}=-132,34522 \\
\mathrm{I}_{1}=79,33886 \\
\mathrm{I}_{2}=53,35408 \\
\mathrm{I}_{3}=38,25015 \\
\mathrm{I}_{\mathrm{i}}=28,59569\end{array}$ \\
\hline & & & & & & & & & & & & & & $\begin{array}{c}13,234699 \\
\mathbf{I}_{0}\end{array}$ & 79,33886 & $\begin{array}{c}53,35408 \\
\mathrm{I}_{2}\end{array}$ & $\begin{array}{c}38,25015 \\
\mathbf{l}_{3}\end{array}$ & $\begin{array}{c}28,59569 \\
\mathrm{I}_{4}\end{array}$ & \\
\hline
\end{tabular}

T'sbetLA 2

\begin{tabular}{|c|c|c|c|c|c|c|c|c|c|c|c|c|c|c|c|c|}
\hline 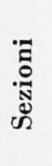 & $\begin{array}{c}x \\
\text { distanza } \\
\text { da } \\
\text { Garda } \\
\text { km }\end{array}$ & $\begin{array}{c}S(x) \\
10^{6} \mathrm{~cm}^{2}\end{array}$ & $\begin{array}{c}b(x) \\
10^{4} \mathrm{~cm}\end{array}$ & $\begin{array}{c}v(x) \\
\text { parziali } \\
6.25 \times \\
\times 10^{4} \mathrm{~m}^{2}\end{array}$ & $\begin{array}{c}(x) \\
6.25 \times \\
\times 10^{4} \mathrm{~m}^{2}\end{array}$ & $\begin{array}{c}\sigma(x)= \\
=S(x) \cdot \bar{b}(x) \\
h^{3} \mathrm{~m}^{3}\end{array}$ & $\left(=\frac{v}{a}\right)$ & $z^{2}$ & $1 \cdot z$ & $(1-\boldsymbol{z})^{2}$ & $z^{2}(1-z)^{2}$ & $\begin{array}{c}\frac{z^{2}(1-z)^{2}}{\sigma(z)} \\
{[\mathrm{hm}]}\end{array}$ & $\triangle z$ & $\begin{array}{c}\frac{z^{2}(1-z)^{2} \triangle z}{\sigma(z)} \\
=M \\
10^{-5}\end{array}$ & $\begin{array}{l}M z \\
10^{-5}\end{array}$ & $\begin{array}{l}M z^{2} \\
10^{-5}\end{array}$ \\
\hline 1 & 0.0 & 0.0 & 0.0 & 0,0 & 0,0 & 一 & 0.00000 & 0.00000 & 1.00000 & 1,00000 & 0.00000 & .- & - & $\ldots$ & & \\
\hline 2 & .125 & 155.0 & $\begin{array}{r}0.0 \\
15.4\end{array}$ & $\begin{array}{l}, 0 \\
2,9\end{array}$ & 2,9 & 23.9 & .00204 & .000004 & .99796 & $\begin{array}{r}1,00000 \\
.99592\end{array}$ & $\begin{array}{r}.00000 \\
.00004\end{array}$ & .0000002 & .00204 & .0000408 & .0000001 & 0 \\
\hline 3 & .375 & 475.0 & 20.8 & 7,3 & 10.2 & 98.8 & .00716 & .000051 & .99283 & .98571 & .00005 & .0000005 & .00512 & .0002560 & .0000018 & .0000000 \\
\hline 4 & .9 & 1057.5 & 25.7 & 18,8 & 29.0 & 271.8 & .02037 & .000415 & .97963 & .95967 & .00040 & .0000015 & .01321 & .0019815 & .0000404 & .0000008 \\
\hline 5 & 1.4 & 1447.5 & 36.5 & 25,7 & 54.7 & 528.3 & .03842 & .001476 & .96158 & .92464 & .00136 & .0000026 & .01805 & .0046930 & .0001803 & .0000069 \\
\hline 6 & 1.9 & 1580.0 & 38.7 & 30,9 & 85.6 & 611.5 & .06013 & .003616 & .93887 & .88336 & .00319 & .0000052 & .02171 & .0112892 & .0006788 & .0000408 \\
\hline 7 & 2.4 & 1655.0 & 40.3 & 25,5 & 111.1 & 667.0 & .07804 & .006090 & .92196 & .85001 & .00518 & .0000078 & .01791 & .0139698 & .0010902 & .0000051 \\
\hline 8 & 2,9 & 1812.5 & 42.2 & 28,4 & 139.5 & 764.9 & .09798 & .009600 & .90202 & .81364 & .00781 & .0000102 & .01994 & .0203388 & .0019928 & .0001952 \\
\hline 9 & 3.4 & 2345.0 & 49.2 & 37,1 & 176.6 & 1153.7 & .12404 & .015386 & .87596 & .76731 & .01181 & .0000102 & .02606 & .0265812 & .0032971 & .0004090 \\
\hline 10 & 3.9 & 3550.0 & 65.5 & 58,2 & 234.8 & 2325.25 & .16492 & .027199 & .83508 & .69736 & .01897 & .6000082 & .04688 & .0335216 & .0055284 & .0009117 \\
\hline 11 & 4.4 & 3707.5 & 68.3 & 54,4 & 289.2 & 2550.8 & .20313 & .041262 & .79687 & .63500 & .02620 & .0000103 & .03821 & .0393563 & .0079944 & .0016239 \\
\hline 12 & 4.9 & 3770.0 & 70.0 & 53,2 & 342.4 & 2639.0 & .24050 & .057840 & .75950 & .57684 & .03336 & .0000126 & .03737 & .0470862 & .0113242 & .0027235 \\
\hline 13 & 5.4 & 3955.0 & 69.0 & 51,2 & 393.6 & 2728.95 & .27646 & .076430 & .72354 & .52351 & .04001 & .0000147 & .03596 & .0528612 & .0146140 & .0040402 \\
\hline 14 & 5.9 & 3765.0 & 70.0 & 49,0 & 442.6 & 2635.5 & .31088 & .096646 & .68912 & .47489 & .04590 & .0000174 & .03442 & .0598908 & .0186188 & .0057882 \\
\hline 15 & 6.4 & 3795.0 & 72.2 & 55,5 & 498.1 & 2740.0 & .34986 & .122402 & .65014 & .42268 & .05174 & .0090189 & .63898 & .0736722 & .0257749 & .0090176 \\
\hline 16 & 6.9 & 3970.0 & 74.0 & 62,2 & 560.3 & 2937.8 & .39355 & .154882 & .60645 & .36778 & .05696 & .0000194 & .04369 & .0847586 & .0333567 & .0131276 \\
\hline 17 & 7.4 & 3910.0 & 76.5 & 65,1 & 625.4 & 2991.15 & .43928 & .192967 & .56072 & .31441 & .06067 & .0000203 & .04573 & .0928319 & .0407792 & .0179135 \\
\hline 18 & 7,9 & 3535.0 & 75.2 & 83,2 & 688.6 & 2658.3 & .48367 & .233937 & .51633 & .26660 & .06237 & .0000235 & .04439 & .1043165 & .0504548 & .0244035 \\
\hline 19 & 8.4 & 3507.5 & 80.5 & 56,4 & 745.0 & 2823.5 & .52328 & .273822 & .47672 & .22726 & .06223 & .0000220 & .03961 & .0871420 & .0455997 & .0238614 \\
\hline 20 & 8.9 & 3017.5 & 74.3 & 60,2 & 805.2 & 2242.0 & .56557 & .319869 & .43443 & .18873 & .06037 & .0000269 & .04229 & .1137601 & .0643393 & .0363883 \\
\hline 21 & 9.4 & 2305.0 & 72.5 & 53,0 & 858.2 & 1671.1 & .60280 & .363368 & .39720 & .15777 & .05733 & .0000343 & .03723 & .1276989 & .0769769 & .0464017 \\
\hline 22 & 9.9 & 2175.0 & 70.5 & 57,8 & 916.0 & 1533.4 & .64339 & .413951 & .35661 & .12717 & .05264 & .0000343 & .04059 & .1392237 & .0895751 & .0576318 \\
\hline 23 & 16.4 & 1815.0 & 88.5 & 54,2 & 970.2 & 1606.3 & .68146 & .464388 & .31854 & .10147 & .04712 & .0000293 & .03807 & .1115451 & .0760135 & .0518002 \\
\hline 24 & 10.9 & 1482.5 & 85.0 & 70,8 & 1041.0 & 1260.1 & .73119 & .534639 & .26881 & .07226 & .03863 & .0000306 & .04973 & .1521738 & .1112680 & .0813580 \\
\hline 25 & 11.4 & 1190.0 & 83.3 & 80,1 & 1121.1 & 991.3 & .78746 & .620093 & .21254 & .04517 & .02801 & .0000282 & .05627 & .1586814 & .1249552 & .0983972 \\
\hline 26 & 11.9 & 912.5 & 81.2 & 82,2 & 1203.3 & 740.95 & .84519 & .714346 & .15481 & .02397 & .01712 & .0000231 & .05773 & .1333563 & .1127114 & .0952625 \\
\hline 27 & 12.4 & 712.5 & 61.2 & 61,0 & 1264.3 & 436.05 & .81804 & .788615 & .11196 & .01254 & .00989 & .0000227 & .04285 & .0972695 & .0863792 & .0767082 \\
\hline 28 & 12.9 & 515.0 & 54.5 & 48,2 & 1312.5 & 280.7 & .92189 & .849881 & .07811 & .00610 & .00518 & .0000184 & .03385 & .0622840 & .0574190 & .0529340 \\
\hline 29 & 13.4 & 270.0 & 40.8 & 40,1 & 1352.6 & 110.2 & .95006 & .902614 & .04994. & .00249 & .00225 & .0000204 & .02817 & .0574668 & .0545969 & .0518703 \\
\hline 30 & 13.9 & 167.5 & 31.5 & 34,4 & 1387.0 & 52.8 & .97422 & .949105 & .02578 & .00066 & .00063 & 0000119 & .02416 & .0287504 & .0280092 & .0272871 \\
\hline 31 & 14.4 & 70.0 & 15.5 & 20,5 & 1407.5 & 10.85 & .98862 & .977370 & .01138 & .00013 & .00013 & .0000120 & .01440 & .0172800 & .0170833 & .0168889 \\
\hline 32 & 14.9 & 25.0 & 9.0 & 10,9 & 1418.4 & 0.225 & .99628 & .992574 & .00372 & .00001 & .00001 & .0000444 & .00766 & .0340104 & $.033883^{\circ}$ & .0337578 \\
\hline 33 & 15.4 & 0.0 & 0.0 & 5,3 & 1423.7 & 0.0 & 1,00000 & 1,000000 & .00000 & .00000 & .00000 & .0000000 & .00372 & 1,9880880 & 1,1945375 & $.830834^{\circ}$ \\
\hline
\end{tabular}


2. - Il primo grosso problema da risolvere consisteva nel rispondere alla seguente domanda: l'oscillazione di $30^{\text {m }}$ interessa l'intero bacino Peschiera-Garda considerato come golfo aperto sul restante bacino occidentale?

Abbiamo già risposto altrove negativamente a questa domanda $\left({ }^{1}\right)$. Qui trattiamo la questione in dettaglio.

Furono fatte due ipotesi. La prima contempla l'oscillazione del bacino come golfo aperto sulla bocca punta S. Vigilio-Sirmone, oscillante in media direzione Est-Ovest e quindi con andamento pressoché parallelo alla costa Peschiera-Lazise-Garda. Furono allora tracciate tredici sezioni del bacino stesso con l'andamento dianzi accennato. Nella Tabella 1 sono contenuti i dati tratti dalla morfologia delle sezioni stesse, nonché i risultati dell'applicazione del metodo Hidaka. La teoria di tale metodo è stata più volte precedentemente esposta.

Nel caso $m=2$ si ottennero i seguenti risultati:

$$
\begin{aligned}
& \text { periodo oscillazione uninodale }=56^{\mathrm{m}} \\
& \text { periodo binodale }=29^{\mathrm{m}}, 5 .
\end{aligned}
$$

Tali risultati sono evidentemente negativi ai fini della nostra ricerca. Va osservato infatti che essi risulterebbero anche maggiori di quelli ottenuti, in quanto andrebbero moltiplicati per il fattore di correzione di hocea, nel caso in esame piuttosto sensibile. Un'oscillazione uninodale di cerlo superiore all'ora, sarebbe possibile solo se il golfo Peschiera-Garda fosse aperto su un bacino con periodo di oscillazione uninodale per lo meno uguale. Sappiamo invece che il Garda nel suo complesso ha un'oscillazione uninodale di $43^{\mathrm{m}}$. Il valore oltenuto per questa via è quindi da scartare. Lo stesso dicasi per l'oscillazione binodale, che risulterebbe notevolmente superiore all'oscillazione binodale dell'intero lago $\left(24^{\mathrm{m}} \mathrm{ca}.\right)$.

Le oscillazioni con le caratteristiche sopraddette potrebbero aver luogo eventualmente solo come oscillazioni forzate.

Siamo allora passati ad una seconda ipotesi, che considera l'intero hacino orientale come oscillante a sé in direzione Nord-Sud.

Il bacino Peschiera-Garda $f u$ suddiviso in 33 sezioni. normali alla linea di valle, con generale andamento Est-Ovest (fig. 1). La linea di valle è costituita dal canale subacqueo che dalla secca del Vò - e sul lato occidentale di questa - conduce a Peschiera, di dove esce con il nome di Mincio.

La Tahella 2 porta le distanze delle singole sezioni contate a partire da Garda, l'area delle sezioni stesse, la loro larghezza in super- 
ficie e gli altri elementi necessari per l'applicazione del metodo di Hidaka. Detto metodo è stato applicato per il caso $m=1$, al quale corrisponde, per i periodi, la seguente equazione:

$$
\left(I_{0} I_{2}-I_{1}{ }^{2}\right) \lambda^{2}-\left(\frac{2}{15} I_{0}-\frac{1}{3} I_{1}+\frac{1}{3} I_{2}\right) \lambda+\frac{1}{60}=0,
$$

dove

$$
I_{\mathbf{n}}=\int_{0}^{1} \frac{z^{2}(1-z)^{2} z^{11}}{\sigma(z)} d z \quad(\mathbf{n}=0,1,2)
$$

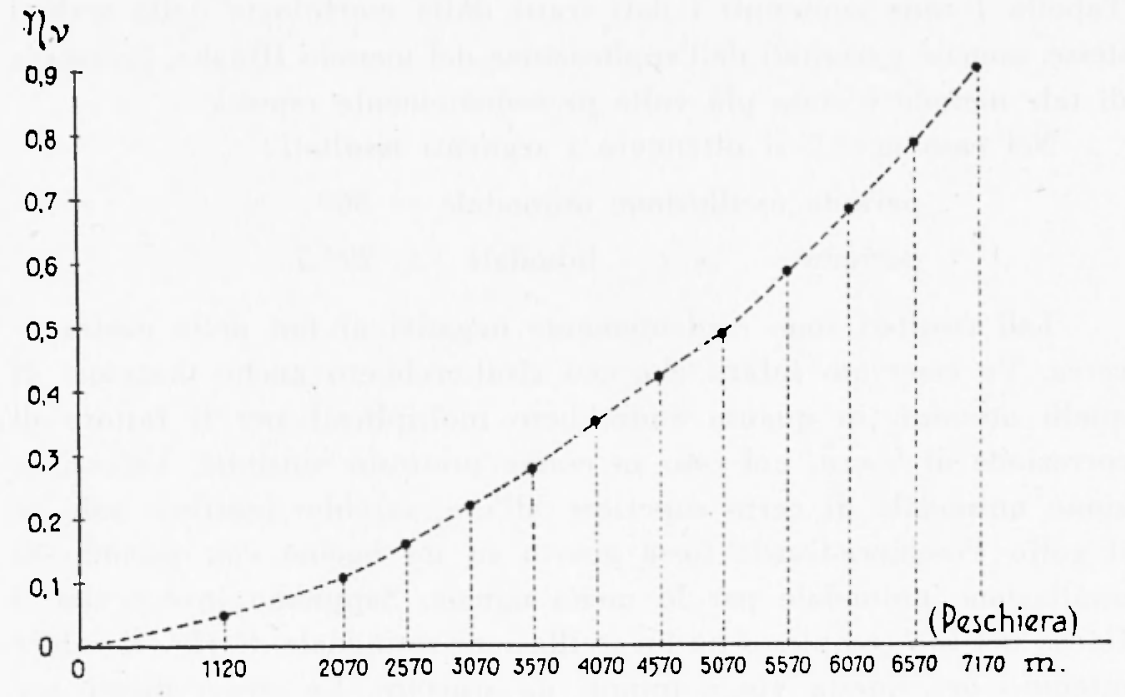

Iig. 2

essendo $z=v / a$ e $v$ l'area, calcolata alla superficie libera, della superficie delimitata da una determinata sezione verticale $S(x)$ e dall'estremo $x=0$; a è l'area superficiale totale del bacino. Si sa inoltre che $\sigma(z)=b(z) S(z)$. Con i dati della Talsella 2 si sono ottenuti $i$ :eguenti valori per $I_{0}, I_{1}, I_{2}: I_{0}=1,988088010^{-5}: I_{1}=1,194537510^{-5}$ : $I_{2}=0,830834910^{\circ}$. L'unità scelta fu l'ettometro. Risolta l'equazione dei periodi si sono ottenuti per $\lambda$ i valori:

$i_{1}=15198 ; \quad i_{2}=48762$, che corrispondono all'oscillazione uninodale e binodale rispettivamente.

A questi valori corrispondono i periodi $T_{1}=24^{\mathrm{m}}, 1 ; T_{2}=13^{\mathrm{m}} .5$. $\mathrm{Va}$ tenuto conto ancle per questo caso, della correzione di bocca. 
A questo riguardo, dolıbiamo però osservare che noi abbiamo consideralo il bacino come oscillante in sé e non come golfo. Pertanto nella formula che dá il termine correltivo per l'influsso dell'apertura all'estremo Nord-Ovest, dobbiamo introdurre in luogo della lunghezza dell'intero golfo, la distanza dall'estremo Sud dell'uninodo.

Passando al calcolo, si trova che l'equazione dell'uninodo è:

$$
6,287+z^{2}-2,1916 z-1=0,
$$

la quale, risolta in $z$, per i valori compresi fra 0 e 1 , conduce a $z=0,6095$. L'uninodo pertanto verreble a trovarsi a cirea $9 \mathrm{~km}$ a Sud di Garda.

Poiché il rapporto fra l'apertura del bacino considerato e la sua lunghezza i dell'ordine di $9 / 10$, ne consegue per il fattore di correzione il valore 1,2. L'uninodale pertanto ha un valore effettivo di:

$$
T=29^{\mathrm{m}} \text { ca. }
$$

Questo risultato poteva essere ritenuto soddisfacente. Esso consentiva infauti di poter concludere che l'oscillazione di $30^{\mathrm{m}}$ ("al. registrala a Peschiera interessara il bacino Peschiera-Garda solo parzialmente: in altri termini l'oscillazione stessa non aveva la linea nodale in corrispondenza del trallo punta Sirmione-punta S. Vigilio, hensì un uninodo interno al bacino stesso. Si poteva quindi arguire che tale uninodo dovera limitare, verso Nord, la parte del bacino PeschieraGarda oscillante come golfo con il periodo suddetto.

3. -- Restava da trovare una conferma sperimentale a questo risultato, conferma che arrebbe servito poi per l'applicazione di un metodo analitico, atto a precisare il valore dell'uninodale del golfo ridotto.

Ci siamo serviti a questo scopo di un modello del lago di Garda, espressimente costruito per lo studio particolaregrgiato delle oscillazioni libere dell'intero lago e di sue singole parti ${ }^{2} \%$.

f) noto che, conosciuto il rapporto fra la scala delle dimensioni orizzontali e quella verticale, grazie al principio di Harris, è possibile risalire dal comportimento del modello a quello effetlivo del bacino naturale. Se indichiamo con a il rapporto fra le dimensioni 
orizzontali del hacino naturale e del modello e con $b$ il rapporto delle corrispondenti dimensioni verticali, il fattore che consente di passare dai periodi osservati sul modello ai reali periodi del bacino, è dato da:

$$
\tau=a / r^{-} \text {. }
$$

Il modello in parola è stato costruito in modo da far assumere a $\tau$ il valore 850 .

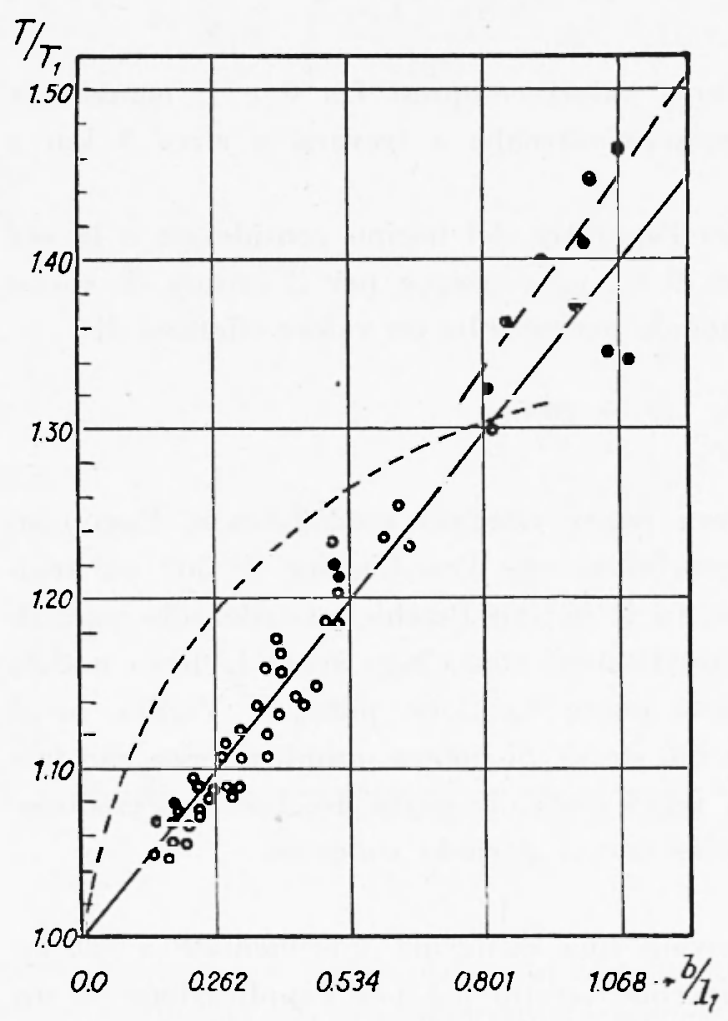

Fig. 3 - Dipendenza del fattore di correzione $T / T_{1}$ dalle dimensioni della bocca e del bacino $\left(b=\right.$. larghezza del bacino; $l_{1}=$ lunghezza effettiva del bacino; la linea curva rappresenta la teoria "giapponese ») i punti e i tratti rettilinei rappresentano i risultati delle esperienze di Neumann).

Per quanto si riferisce al hacino Peschiera-Garda, furono seguiti opportuni accorgimenti che hanno consentito di ottenere, come periodo della oscillazione fondamentale del relativo modello, il valore $T=2^{\mathrm{s}}, \mathbf{0 5}$. Che questo sia il periodo della fondamentale del golfo ridotto Peschiera-Garda ì provato dal fatto che, con tale ritmo, l'oscillazione del modello persiste a lungo, presentando la linea nodale in corrispondenza del tratto punta Sirmione Lazise.

Tenulo conto del rapporto di riduzione con cui $\dot{c}$ stato costruito il modello, ne segue che il periodo della fondamentale del hacino Peschiera - Garda deve essere dell'ordine di $29^{\mathrm{m}}$ ca. Questo valore è assai prossimo alla media dei periodi di detta oscillazione, quali vengono osservati a Peschiera. 
4. - Si aveva cosi la prova sperimentale sull'esistenza di una oscillazione che interessava solo parzialmente il bacino Peschiera-Garda, nel senso che la sua linea nodale può essere senz'altro ritenuta come limite della parte del bacino che entra in oscillazione con quel periodo.
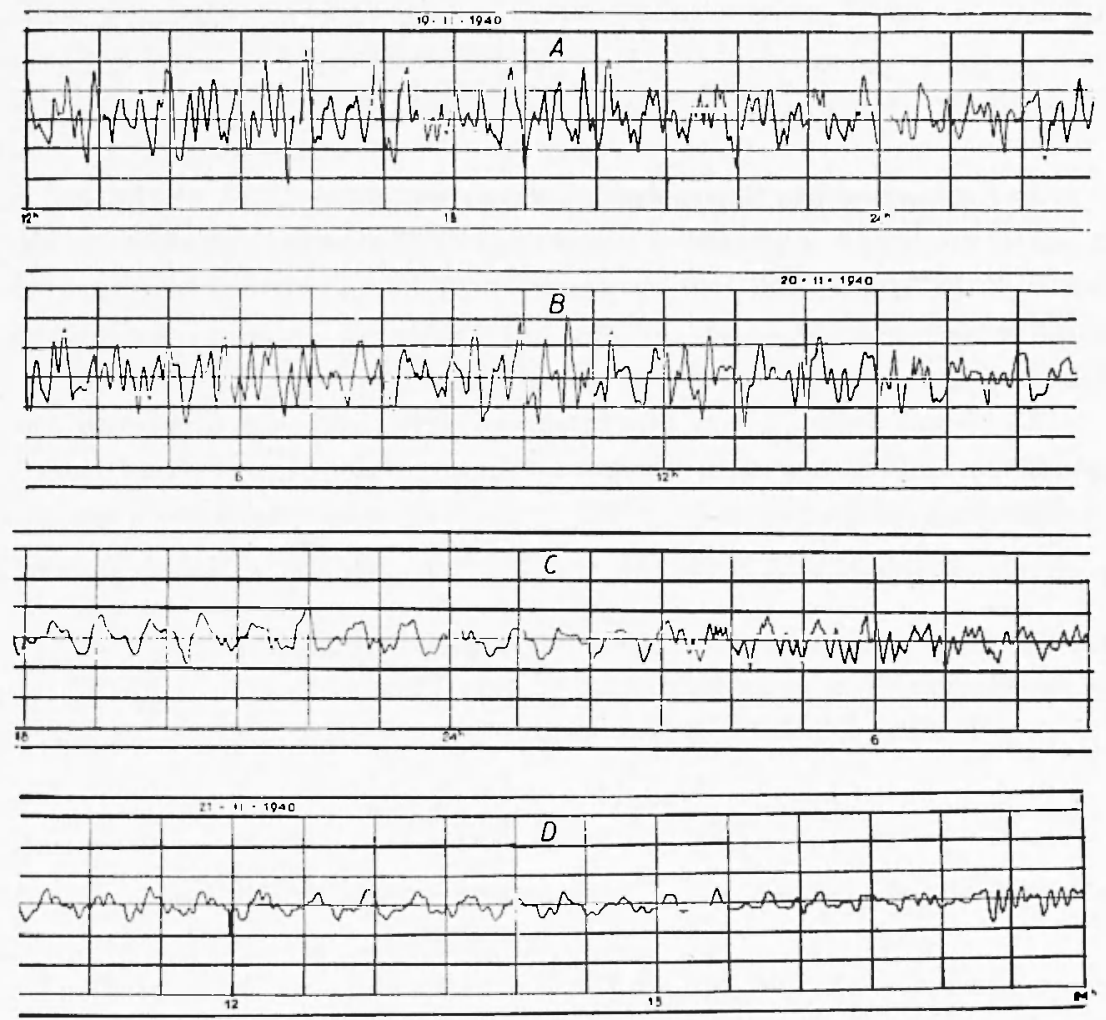

Fig. 4 (Riduzione $1 / 17$ dell'ampiezza naturale).

Una ulteriore conferma di questo risultato potera aversi applicando al grolfo ridotto uno dei metodi analitici che valgono appunto per i bacini aperti. Ed è quello che è stato fatto con l'applicazione del metodo di Goldberg.

Tale metodo è stato esposto in un lavoro precedente, al quale rimandiamo il lettore $\left({ }^{3}\right)$. Riassumiamo qui soltanto quella parte che ci è servita per i calcoli.

Indichiamo con $\xi_{v}$ ed $\eta_{r}$ oli spostamenti massimi orizzontali e verticali determinati nell'interno del golfo, in corrispondenza della sezione $r$ ma, dal passagrgio della massa d'acqua arbitraria $m_{0}$, che 
attraversa la sezione di bocca nel tempo pari ad un quarto di periodo. Dalle equazioni di moto e di continuita dell'idrodinamica, i trae allora, nel caso di moto sinusoidale:

$$
\begin{gathered}
\frac{d \eta_{v}}{d x}=\frac{4 \pi^{2}}{g T^{*}} \cdot \xi_{v} \\
\eta_{v}=-\frac{1}{b(x)} \cdot \frac{d}{d x}\left\{g\left(x_{v}\right) \cdot E_{,}\right] .
\end{gathered}
$$

Il hacino va inteso suddiviso, a partire dalla linea di bocra, in $n$ parti, mediante $n$ sezioni trasversali $S v$, praticate normalmente alla linea di valle nei punti $x=x_{v}(v=1,2,3, \ldots . n)$, alle distanze rispettive $\Delta x_{v}$ anche esse contate sulla linea di valle. Si suole indicare con $\Delta v_{v}$, le porzioni di superficie libera comprese fra $S_{v}$, ed $S_{1-1}$

La prima delle [1] da l'inclinazione della tangente al profilo longritudinale della superficie libera oscillante del golfo. Poiché questa inclinazione viene supposta costanle per ogni suddivisione (cio) che equivale a ritenere trascurabile l'ammontare di $\frac{d^{2} ! !}{d x^{\prime \prime}}$, cone generalmente si verifica nei hacini naturali il profilo longitudinale del golfo in oscillazione viene approssimato ad ma linea spezzata. Si ha:

$$
\Delta \eta_{\gamma_{+1}}=\frac{d \eta_{v}}{d x} \Delta x_{v+1}
$$

e lo schema di calcolo per l'interrazione numerica diviene:

$$
\begin{aligned}
& \xi_{v}=\frac{m_{v}}{s_{v}}, \quad \frac{d \eta_{v}}{d x}=\frac{4 \pi^{2}}{g T^{2}} \cdot \xi_{v} \\
& \eta_{v+1}=\eta_{v}+\frac{d \eta_{-}}{d x} \cdot \Delta x_{v+1}, m_{v+1}=m_{v}-\frac{\eta_{v}+\eta_{v-1}}{2}
\end{aligned}
$$

L'ultima delle [2] sta ad indicare che $\mathrm{il}$ dislivello sulla superficie $v+1$ che intercede fra le sezioni $S_{v}$ ed $S_{S_{-1}}$ ¿ dovuto all'eccedenza dell'acqua entrante da $S$ su quella uscente da $S_{;}-$. $_{\text {. }}$

Il periodo prescelto potrà allora ritenersi preciso quando, all'estremo chiuso del golfo, si abbia.

$$
m_{0}-\frac{n-1}{1} \frac{\eta_{v}+\eta_{1} \div 1}{2} \Delta v_{1+1}=0
$$


Sensibili scostamenti, in un senso o nell'altro, da questa condizione comportano un'opportuna variazione del periodo prescelto.

Per quanto riguarda il caso in esame, furono praticate al golfo ridotto, a partire dalla linea nodale, considerata come linea di hocca, dodici sezioni. La Tabella 3 riporta nelle prime quattro colonne i dati necessari all'applicazione del metodo, e cioc il numero d'ordine delle sezioni, l'area delle sezioni trasversali, la distanza tra una sezione e l'altra e l'area delle porzioni di superficie dell'acqua, comprese fra due sezioni conseculive.

Furono fatte applicazioni del metodo partendo da un valore di 2.9", valore che si i dimostrato troppo piccolo.

Per un periodo di $25^{\prime \prime}$ veniva invece un piccolo eccesso nella condizione di azzeramento contenuta nella [3]. Cina ulteriore applicazione fatta per $T=23^{m}: 8$ la portato a soddisfare in pieno la [3] I'Tabella 3 e fig. 2 \%.

Restava ora da stabilire la correzione di hocea. E noto che i giappenesi Honda, Terada, Yoshida e Isilani f', hanno trovato, come fattore correltivo, dovuto allapertura dei golfi, l'espressione:

$$
(1+4 P b / l)^{1 / 2}
$$

dove $b$ i la larghezza della bocra. $l$ la lunghezza del golfo e

$$
P=\frac{1}{2 \pi}\left(3 / 2-\gamma-\log _{\mathrm{e}} \frac{\pi l}{4 \cdot l}\right),
$$

essendo $\gamma$ la costante di Mascheroni $(\gamma=0,5772 \ldots .$.$) .$

Nel nostro caso il rapporto $b / l$ vale 0,8 . Per un tale rapporto si otliene un fattore di correzione pari ad 1,3. Pertanto il periodo del. l'oscillazione fondamentale del grolfo ridotto risulta. in definitiva.pari a

$$
T=30^{\mathrm{m}}, 9 \text {. }
$$

Si sa pero che la formula ottenuta dai wiapponesi non è da tutti accetlata come rispondente alla realta dei fatti. Comunque essa vale per hacini aperti di forma reliangolare e con profondita uniforme. Di recente la questione i stata ripresa dal tedesco G. Neumann ("), il quale ha conclueo che risponte molto meglio allesperienza il considerare un fattore correttivo cresconte linearmente al crescere del rapporto $b / l$. 


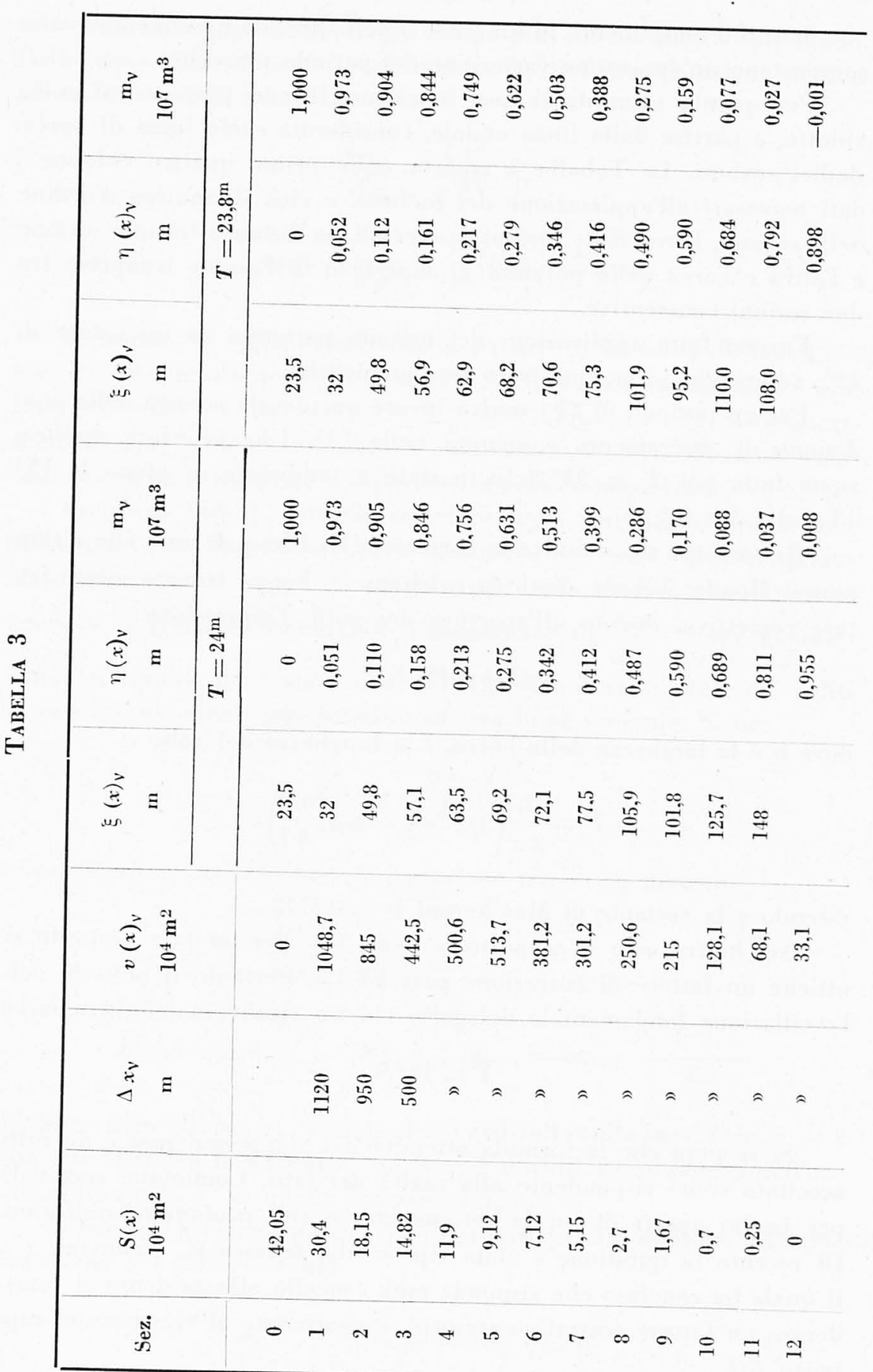



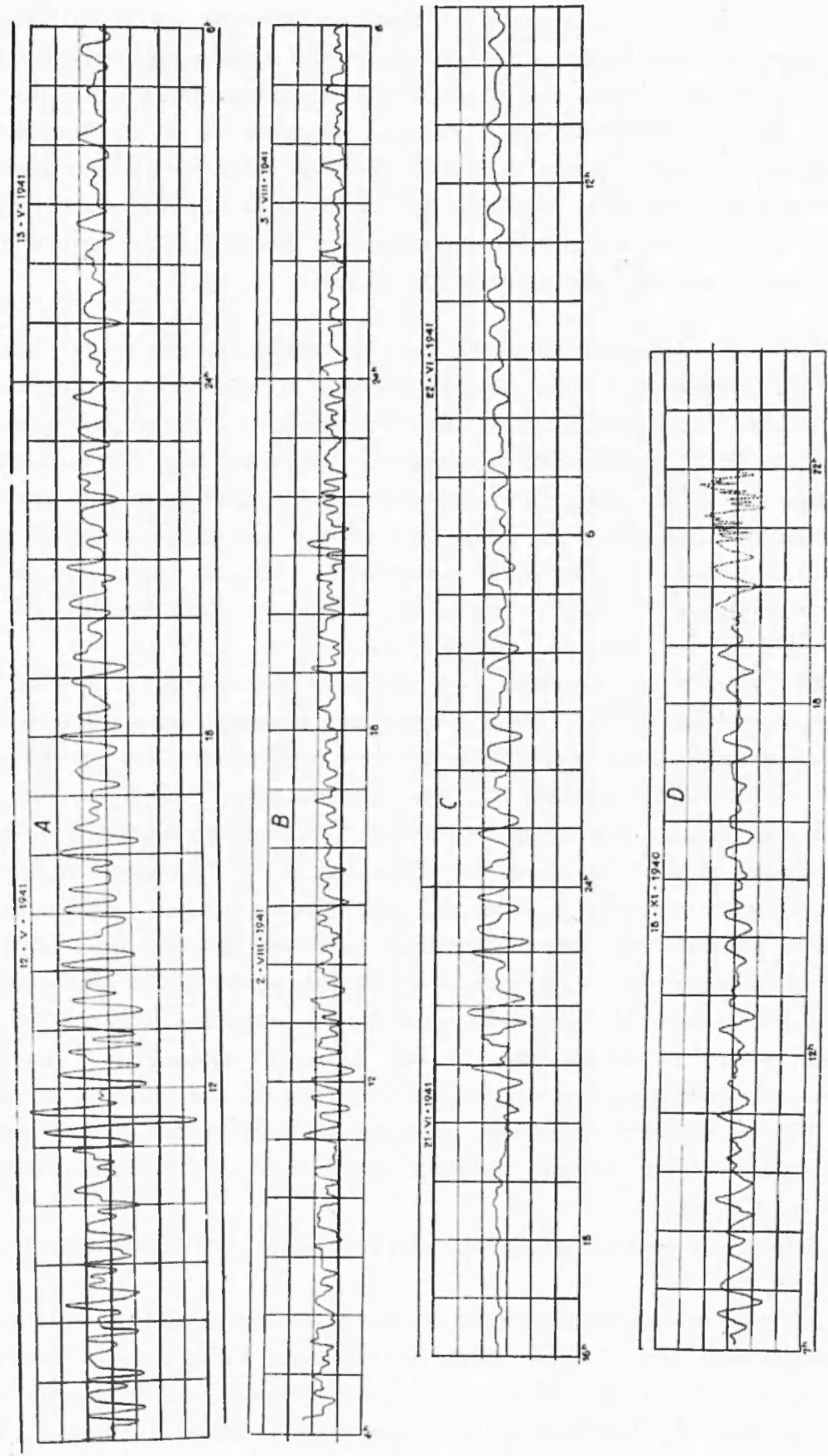

Fig. 5 (Riduzione $1 / 7$ dellampiezza naturale). 
Nella fig. 3 sono riportati i risultati oltenuti sperimentalmente da Neumann, nonché la curva corrispondente alla formula griapponese. Da detla figura risulta che il divario i particolarmente notevole per valori del rapporto $b / l$ minori di 0,5 e maggiori di 1 . Per yuanto si riferisce al caso in esame sia la formula grapponese sia la relazione di Neumann conducono allo stesso risultato, come appare chiaro dalla figura cilata. Pertanto possiamo concludere assegnando al valore ottenuto per il periodo una buona attendibilita.

5. - Registrazioni sistematiche dei movimenti liberi del hacino Peschiera-Garda non sono ancora state compiute. Però a Peschiera, da qualche decennio, funziona un idrometrografo, il cui scopo specifico è quello di rivelare le variazıoni di livello del lago. Lo strumento ha una sensibilità sufficiente per consentirgli altresi la regristrazione delle sesse. Esso infatti dà quasi di continuo notevoli esempi di registrazione delle sesse uninodali interessanti l'intero lago, nonché di altri movimenti liberi che riguardano sia il lago nella sua totalità sia il hacino Peschiera-Garda o parti di esso.

Fra queste registrazioni non mancano naturalmente quelle oggretto di questo studio. Nello spoglio dei limnogrammi ottenuti nel 1941 abbiamo trovato evidenti esempi di oscillazioni con periodo fra i $30^{\mathrm{m}}$ e i $31^{\%}$. Nelle figg. $4-7$ ne sono riportati alcuni.

11 modo come si verificano, conferma l'origine idrodinamica di delle oscillazioni, le quali, come appare dalle figg. 5,6 e 7 vengono registrate singolarmente separate da intervalli più o meno lunghi. Ciò si spiega, tenendo presente che tali registrazioni interessano solo una parte del golfo, e quindi sono oscillazioni di un hacino aperto sovra altro bacino molıo più esteso. In tal modo, quando la causa perturbante agrisce, l'oscillazione tornando dalla base del golfo, prosegue oltre l'uninodo come onda progressiva e pertanto si esaurisce in un periodo. L'insorgere di una nuova oscillazione, presuppone la ripresa o la continuità della causa origine (esempi notevoli specie in A, C. D fig. 5 : in A, B fig. 6 e in A fig. 7).

A questo riguardo, gli esempi riportati sono particolarmente significativi.

A Peschiera naturalmente, si ha la registrazione di tutta una serie di oscillazioni che, dall'uninodale interessante l'intero lago (con un periodo di $43^{\mathrm{m}}$ ca. - figr. 6-C) vanno a quelle riguardanti lo zoccolo che dalla spiagrgia di Peschiera si protende verso il largo. Le figrg. 4. 5, 6 riportano esempi anche di queste ultime oscillazioni. Particolarmente 

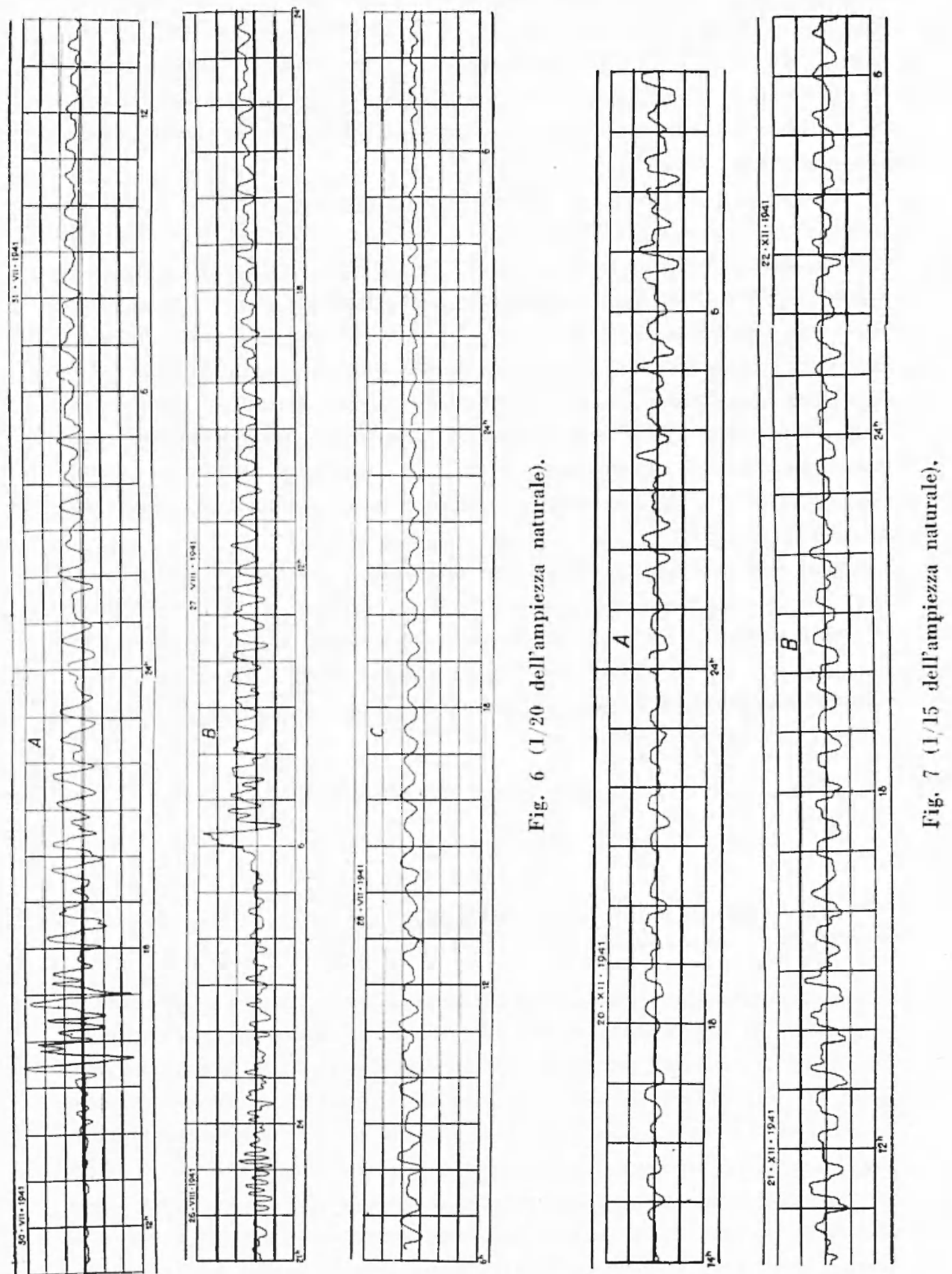
frequenti, si presentano pure oscillazioni libere con periodi propri dell'ordine di 22-24" (fig. 5-A; fig. 6-B). La loro persistenza lasciava prevedere che tali oscillazioni interessavano l'intero lago. La riprova si è aruta con il modello: sperimentando infatti con periodi dellordine di $1,6-1,7^{\mathrm{s}}$, si è constatalo che le oscillazioni mettevano in moto l'intera massa liquida.

Per quanto concerne le cause, che danno origine ai vari tipi di oscillazione registrati a Peschicra, siamo del parere che la più efficiente sia quella legala al transio di perturlazioni microbariche prorenienti da Nord-Ovest. Quando dette perturbazioni si propagano verso Peschiera con velocità dell'ordine di quelle che caratterizzano le onde lihere del lago sottostante, alla hase del golfo (cioè a Peschierai si verificano spostamenti in senso verticale lanto più ampli quanto più detta condizione viene rispettata. Cio è stato già rigorosamente verificato con le sesse del golfo di Trieste ("). Anche negli esempi riportati vi sono casi che possono senzaltro essere attribuiti alla suddelta origine (figr. 6-B).

Ad ogni modo, il risultato più notevole della ricerca è quello di aver provalo, ancora una volta, che l'oscillazione di $30^{\mathrm{m}}$ ca. non $\dot{e}$ affatto la binodale interessante l'intero lago, come aveva supposto Defant, bensi un'oscillazione che riguarda sia a Nord (Riva) che a Sud (Peschieral porzioni limitate di lago, oscillanti con le caratteristiche proprie dei hacini aperti.

Roma - Istituto Nazionale di Geofisica - Ottobre 1953.

\section{RIASSUNTO}

Si riportano $i$ risultati di una lunga ricerca, intesa a spiegare il complesso di oscillazioni libere che vengono registrate a Peschiera, alla base del bacino orientale del lago di Garda. Fra queste oscillazioni, di particolare interesse si presentava quella avente un periodo di $30^{\mathrm{m}}$ ca. La spiegazione fisica di questa sessa era rimasta controversa per oltre un quaranlennio. Questa ricerca ha permesso di chiarire che tale oscillazione non è afjatto la binodale dell'intero lago, come sosteneva Defant, ma interessa solo una parte del golfo di PeschieraGarda, del quale costituisce l'oscillazione fondamentale. Ciò in analogia con la corrispondente oscillazione, di ugual pcriodo, che muove la 
parte settentrionale del Garla, come provano le registrazioni ottenute a Riva.

Alcune considerazioni finali si riferiscono alla probabile origine delle oscillazioni libere che vengono registrate "Peschiera.

\section{SLIMMARY}

This report concerns the results of an extensive research, intended (1) explain the complex of free oscillations which are registered at Peschiera, at the base of the eastern basin of the Lake of Garda. Among these oscillations, there ats one of particular interest uhich had a period of about $.30^{m}$. The physical explanation of this seiche has remained controversial for more than forty years. This research has shoun clearly that such an oscillation is not at alt bi-nodal in the uhole late as maintained by Defant, but concerns only a part of the gulf of Peschiera-Garda, from which these fundamental oscillation is formed. This is analogous to the corresponding oscillation, of equal period, "hich moves the northern part of Garda, as is shown by the registrations obtained at Riva.

Some final considerations refer to te probable origin of the free oscillations uhich are registered at Peschiera.

\section{BIBLIOGR AFIA}

(1) C.1.ou P. . S.unt M. C., Su una questione di idrodinamica concernente il lago di Garda. Rend. Acc. Niz. Lineei, Classe Sc. fis. mat. e nat., serie VIII, vol. XIV, fasc. 3 (1953).

(-) Calor P., Le sesse del lago di Garda. Parte III. Prove con il modello. Annali di Geofisicil, II 11949 .

(3) Calor P., Le sesse del lago di Garda. Parte II. Annali di Geofisica, I (1948).

(4) Honda K., Terma T., Yosmm Y., Istraxi D., An investigation on the se. condary Lndulations of oceanic Tides. The Journal of the College of Science, XXIY 11908 ).

(i) Netwax G., Uber Resonanzschwingungen von Meeresbuchen und die Mïndungskorrehtur bei Sciches. Deutsch. Hydrograph. Zeitschrift. I (2/3), 1948.

(ii) Cator P., Sosse dellallo Adriatico, con particolare riguardo "l Gollo di Trieste. Com. Tass. lt., Nem. CCXIVII 11938). 